\title{
OntoFlow, a Software Tool for Surgical Workflow Recording
}

\author{
Dénes Á. Nagy*†, Imre J. Rudas* and Tamás Haidegger*† \\ *Antal Bejczy Center for Intelligent Robotics, EKIK \\ Óbuda University, Budapest, Hungary \\ ${ }^{\dagger}$ Austrian Center for Medical Innovation and Technology (ACMIT) \\ Wiener Neustadt, Austria \\ Email:\{denes.nagy, imre.rudas, tamas.haidegger\}@irob.uni-obuda.hu
}

\begin{abstract}
Surgical Process Modeling is a growing field of biomedical data science, aiming to create and support context aware surgical systems. As a part of it, novel research intends to provide standardized, formal description of surgical processes. Surgical workflow recordings based on ontologies can provide objective measurements of surgical skill, thus standardizing surgical performance. Comparing the operational phase to the calculated optimal process could allow for new, context aware surgical training, evaluation and assistant systems. In this paper, we present a new software tool, named OntoFlow, developed to record ontology-based surgical workflow during the clinical practice, with post-event editing and reviewing capabilities. OntoFlow directly accesses the background ontology, therefore it can speed up the process of ontology development. As a surgical workflow reviewing software it can also be used as a training tool for surgical residents.
\end{abstract}

Index Terms-Surgical Process Modeling; Surgical Data Science; Ontology; Computer-Assisted Surgery; Surgical Workflow

\section{INTRODUCTION}

While surgery used to be a field restricted to medical professionals, with the emergence of Computer-Integrated Surgery (CIS), surgical practice has become an interdisciplinary field [1].

CIS means that a wast amount of information is available pre-operatively (patient specific imaging), during the operation (patient monitoring, guidance systems) and for post-operative evaluation (see Fig. 1). Today, these information sources are represented to the surgeons as individual outputs from separate systems, and the processing of this information is left to the human operators (surgeons, anesthesiologists, technicians etc.). The growing number of these information threads increases the cognitive load on the surgeon, and emphasizes the need for context aware assistant systems capable of filtering out the relevant information during the intervention. Such system needs a higher level understanding of the Surgical Process (SP), so it is able to follow the course of the surgery.

Surgical Process Models (SPMs), are intended to represent the SP, and therefore can be used as a reference frame when storing and analising the recorded surgical data.

The field of process modeling initiates from the need to streamline business processes. For business process modeling, two approaches where developed to create process models: the model either comes from a high level understanding

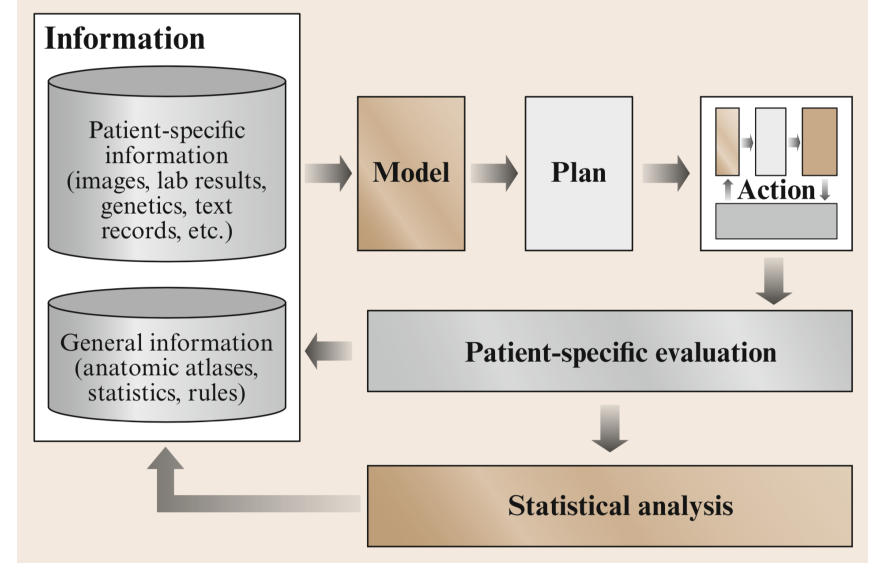

Fig. 1: The concept of Computer-Integrated Surgery (CIS) [2]. Patient specific digital preoperative imaging ( e.g., X-ray imaging, Ultrasound, MR, CT) allows to create accurate preoperative surgical plans. During the procedure, this plan is updated based on available intra-operative sensory information (patient monitoring, image guidance etc.). Post operatively the recorded data can be used for statistical analysis. Surgical workflow recording systems are able to provide the contextual background for both the planned and the recorded information, allowing for process analysis and optimalisation.

of the process, or by recording and analising the process events. For the second option, event logs are processed using mining techniques to reconstruct the process models [3], [4]. Unfortunately, these mining techniques are not directly transferrable to surgery, as processes are not single threaded, and more importantly, accurate process recordings are not available yet, as procedure documentation is mainly paper based, and document's level of detail shows significant variability [5].

To increase the accuracy and reproducibility of surgical procedure descriptions, workflow recording techniques are under development. It will probably take some time to record a critical amount of data, necessary to use the recorded workflows as the basis for a context aware automated surgical assistant systems; However, these SPMs have immediate applications as well:

- Optimizing the Surgical Workflow, and reducing Operat- 

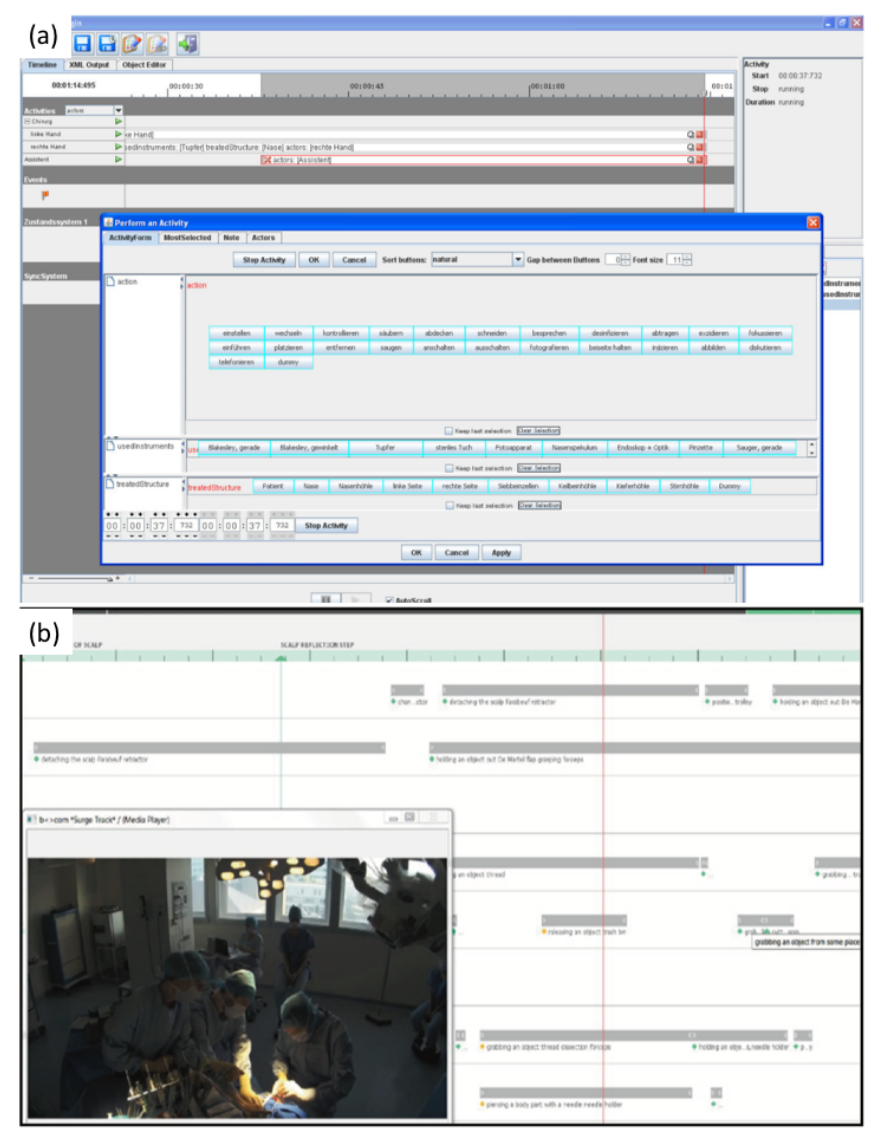

Fig. 2: The two existing surgical Workflow recording tools. (a) The Scientific Workflow Analysis (SWAN) Suite, developed by ICCAS, Universitat Leipzig.

(b) $b<>$ com's Surge Track recording software

ing Room (OR) time;

- Minimizing the occurrence of complications by identifying critical steps in the surgical process;

- Using annotated data to train surgeons;

- Using annotated data to develop and train new context aware systems.

\section{A. Surgical Workflow Recording Tools}

Two centers have developed surgical workflow recording tools so far. The SWAN Suite developed originally at the Innovation Center Computer-Assisted Surgery (ICCAS, Universitat Leipzig) is a software framework created originally as a workflow editor to be used for live recording of surgeries. This Editor was later extended with 3 other components:(1) An ontology based knowledge base for instantiating the concepts used for the workflow recording (2) a visualization tool for graphical representation of the SPM and (3) a reporting tool to the extract quantitative data from the recorded SPM [6]. The other software solution was developed at the University of Rennes and the Institute of Research and Technology (IRT) $b<>$ com [7]. This software has a similar structure to the SWAN suite as it uses an ontological background for instantiating the workflow concepts, and is composed of an editor and an analytics part. B $<>$ com's Surge Track software can be run on Linux, Mac and MS Windows. While both solutions emphasize to build their recordings on an ontological background, experience with the software showed that OWL based ontology integration is not yet fully implemented. Furthermore, these softwares can only be used "as is", and could not be integrated as module to a more complex surgical system.

\section{B. Ontologies}

While SPMs create an organized model for the workflow, SPMs recorded at different centers have different vocabularies, therefore the recorded data is rarely comparable. This is not a new problem to medicine, and on many fields terminology standards already exists. One of the first developed, and internationally accepted standard was the definition of anatomical nomenclatures (such as the Paris anatomical nomenclature (P.N.A.) [8]). These standars can be organized to form an ontological knowledge base, which is easily readable by both humans and machines (Foundational Model of Anatomy [9], SNOMED-CT [10] etc.).

In the field of interventional medicine, sub-domain ontologies are already established [11], [12], [13]:

- Surgical Ontologies for Computer-Assisted Surgery (SOCAS);

- Core ontology for Computer-Assisted Surgery (COCAS);

- Functional Endoscopic Sinus Surgery (FessOnt);

- Ontology for laparoscopic surgeries (LapOntoSPM);

- etc.

To create a high level core ontology connecting the above mentioned sub-domain ontologies to the upper level ontologies (such as the Basic Formal Ontology: BFO, or the Suggested Upper Merged Ontology: SUMO), the OntoSPM international group was formed. Their ontology connects BFO to the subdomain ontologies such as LapOntoSPM [14]. The ontology is intended to be used for simulation-based surgical training, surgical video annotation and surgical workflow management.

\section{Materials \& Methods}

Surgical system development is supported by a growing number of open-source research platforms [15]. These platforms allow for rapid prototyping and development of novel surgical applications. The existing workflow editor softwares are distributed as standalone commercial products, therefore they are not easily integratabtle into newly developed surgical systems. Another issue with these editors is, while they use ontologies as a backend knowledge base, full support for different ontology formats is not achieved.

To provide access to the wides range of ontologies, and not to be restricted to a limited number of ontology formats, we decided to build our program (OntoFlow) as a plugin to the Protégé software. Protégé is an ontology editor originally developed by Mark Musen in 1987, and since then it has become the most often employed ontology editor [16], [17]. It is currently maintained by Stanford's Center for Biomedical 


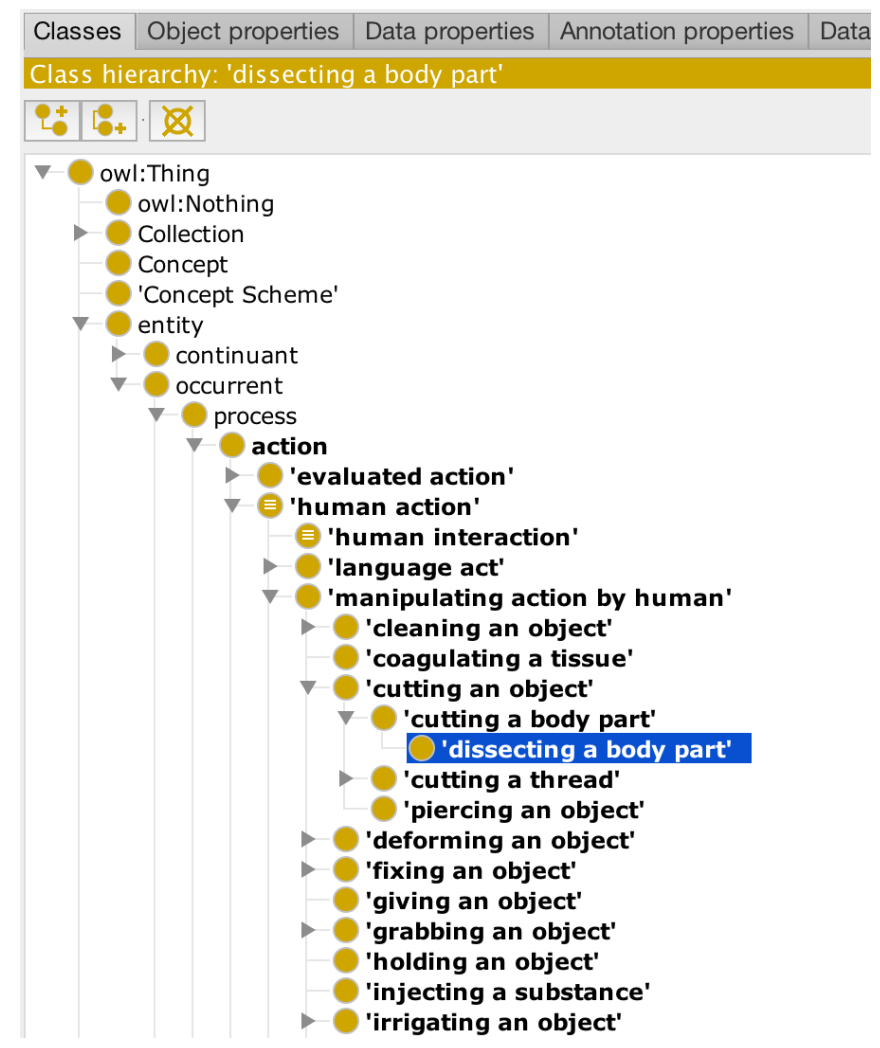

Fig. 3: The ontology explorer provided by Protégé. From the explorer we can select the ontological term, to be added to the workflow.

Informatics Research, and distributed as a free, open source software under the FreeBSD license.

Protégé is built on a modular architecture, and functionalities are added as plugins. It uses the OSGi framework, trough which a plugin has access to both the ontology and the program's main event loop. OntoFlow extends Protégé by allowing the user to record and edit workflows based on the available ontology. OntoFlow's main component is the workflow editor panel (Fig. 4). This panel displays the currently selected workflow element, and allows the modification of it's main properties. these properties define it's name, place in the SP (start and end times) and allow for quick note taking. The program is built to record workflows on a hierarchical manner, where finer granularity level SPMs are encapsulated by higher level concepts. In this way the top level workflow element is the procedure itself (for example: Laparoscopic Cholecystectomy). This element then can be expanded to record the elements building up the procedure workflow. But it is also allowed to expand the elements described here, and record their building elements, and so on. This structure is shown on Fig. 5. The editor panel gives access to this functionality by providing the "Expand" option. We can step back to any of the more abstract levels, by selecting the desired level on the Path. New workflow elements can be added at any abstraction level, as long as the newly added term fits into the

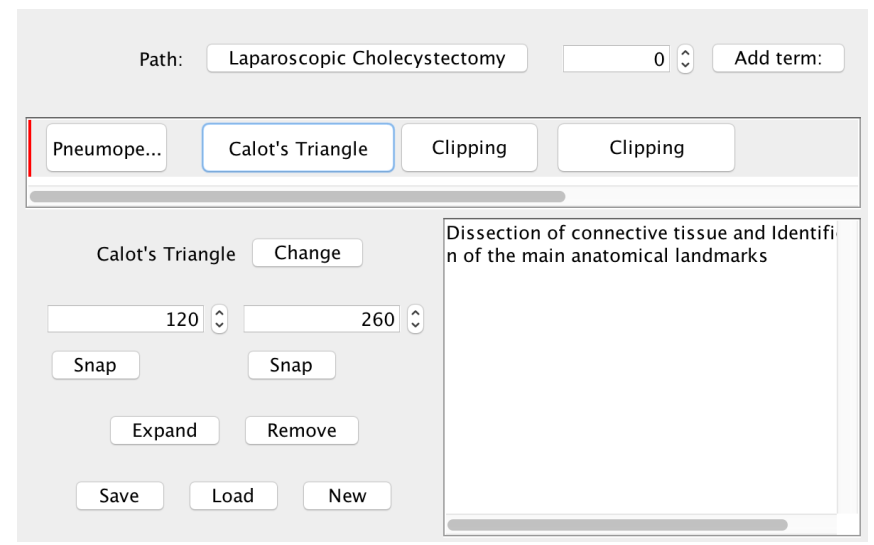

Fig. 4: The OntoFlow panel. The panel is divided into a timeline on the top of the window, and an editor part on the bottom. The timeline shows all workflow elements of the current workflow path (top row of the panel). The editor panel allows the modification (Name, start and stop time, notes) of the selected workflow element. The bottom part of the panel provides functionality to load and save workflows. The Expand button allows to edit the sub elements of the current workflow element.

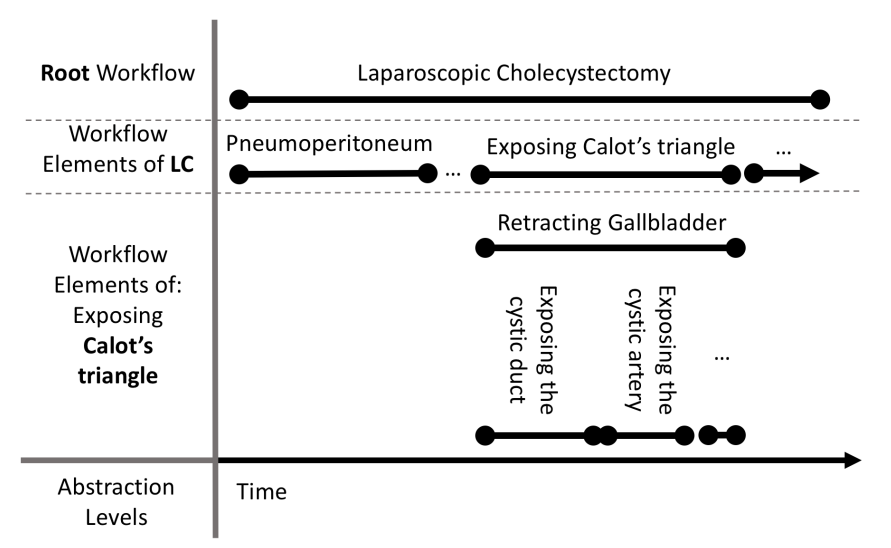

Fig. 5: Sample structure of the workflow recording of the Laparoscopic Cholecystectomy procedure.

timeframe of the parent (encapsulating) workflow element. For example we record a surgical procedure we can not add any element outside of the timeframe of that procedure. Similarly we can not add a sub-element to a procedure step outside of that step's timeframe. The workflow is stored in an XML format because it inherently supports the hierarchical structure the workflow is recorded in, and it allows for a flexible way of building up the multi level SPM.

\section{A. Offline and Online Workflow recording}

When working offline, the plugin loads a video viewer into Protégé. While the editor panel is not dependent on the viewer itself, currently the plugin loads a VLC module into Protégé. We choose VLC because it's free, open source, and supports almost every video formats [18]. The VLC module's time 
is synchronized with the editor panel's time, therefore, all modifications are placed exactly at the desired timepoint on the video.

For online recording, OntoFlow provides a continuous timeline and workflow elements can be added on the fly. As a convenience function online recording adds the new workflow element directly, as the continuation of the previous element (prev.stop_time current.start_time). While in the case of preexisting workflow models it would be beneficial to provide the user with suggested next workflow element choices, it is currently not implemented, and remains the user's task to select the next workflow element directly from the ontology explorer (Fig. 3).

\section{RESUlTS \& DisCUSSION}

OntoFlow works as a plugin following the OSGi structure, and seamlessly integrates into the Protégé architecture. It uses the available ontologies as standard dictionaries to create workflows. Protégé's architecture provides both access to the ontology, and may be used to refine the ontology. This second function, however, will not be necessary if the ontology is finalized. In such a case, one should consider restricting the Protégé user interface, or to use the plugin as a standalone program, in which case read only access to the ontology should be provided using the same OSGi event loops as Protégé.

While OntoFlow was developed with the main intention to record OR workflows, it can also be used to annotate other types of processes, where the main intention is to use an ontological background as building fundation for the process model (medical diagnostic processes, assembly line processes etc.).

OntoFlow allows the recording of SPMs at several granularity levels. As of now, the program does not load data from external sources. For research applications, this integration could be done by either implementing a bridge to the Robot Operating System (ROS) [19] or to OpenIGT [20].

Surgical training is one of the prime areas where this tool can be deployed. With OntoFlow, surgical students can watch recordings of annotated procedures, or annotate new recordings themselves. These recordings are not restricted to video, but can include other channels such as the patient monitor, or even data from a surgical robot. Such practice applied to the medical curriculum would create important databases, which could lead to data mining algorithms.

Real-time integration with external data sources (surgical robot, patient monitor etc.) in a longer term could enable the system to automatically recognize workflow steps, and eventually to create decision support systems, and context aware automation [21].

\section{ACKNOWLEDGMENT}

The research was supported by the Hungarian OTKA PD 116121 grant. T. Haidegger is a MTA Boyai Fellow. He is supported through the New National Excellence Program of the Ministry of Human Capacities. This work has been partially supported by ACMIT (Austrian Center for Medical Innovation and Technology), which is funded within the scope of the COMET (Competence Centers for Excellent Technologies) program of the Austrian Government. We acknowledge the financial support of this work by the Hungarian State and the European Union under the EFOP-3.6.1-16-2016-00010 project.

\section{REFERENCES}

[1] Á. Takács, D. Á. Nagy, I. Rudas, and T. Haidegger, "Origins of Surgical Robotics: From Space to the Operating Room," ACTA POLYTECHNICA HUNGARICA, vol. 13, no. 1, pp. 13-30, 2016.

[2] R. H. Taylor, A. Menciassi, G. Fichtinger, and P. Dario, "Medical robots and systems ch.52," in Springer Handbook of Robotics. Berlin: Springer, 2008, pp. 1199-1218.

[3] W. M. P. van der Aalst, B. F. van Dongen, J. Herbst, L. Maruster, G. Schimm, and A. J. M. M. Weijters, "Workflow mining: A survey of issues and approaches," Data \& Knowledge Engineering, vol. 47, no. 2, pp. 237-267, Nov. 2003.

[4] G. Schimm, "Mining exact models of concurrent workflows," Computers in Industry, vol. 53, no. 3, pp. 265-281, Apr. 2004.

[5] T. Neumuth, P. Jannin, J. Schlomberg, J. Meixensberger, P. Wiedemann, and O. Burgert, "Analysis of surgical intervention populations using generic surgical process models," International Journal of Computer Assisted Radiology and Surgery, vol. 6, no. 1, pp. 59-71, Jan. 2011.

[6] T. Neumuth, N. Durstewitz, M. Fischer, G. Strauss, A. Dietz, J. Meixensberger, P. Jannin, K. Cleary, H. Lemke, and O. Burgert, "Structured recording of intraoperative surgical workflows," in 31th. SPIE - The International Society for Optical Engineering, vol. 7. Horii SC, Ratib OM (eds): SPIE Bellingham, 2006.

[7] $\mathrm{b}<>$ com, "Surgical Workflow toolbox-annotate," https://bcom.com/sites/default/files/Surgery\%20Workflow\%20toolboxannotate.pdf, Dec. 2017.

[8] N. Lotric, "The Paris anatomical nomenclature (P.N.A.) and its changes in stomatology," Stomatoloski Glasnik Srbije, vol. 16, no. 1, pp. 64-66, Feb. 1969.

[9] C. Rosse and J. L. V. Mejino, "A reference ontology for biomedical informatics: The Foundational Model of Anatomy," Journal of Biomedical Informatics, vol. 36, no. 6, pp. 478-500, Dec. 2003.

[10] D. Lee, N. de Keizer, F. Lau, and R. Cornet, "Literature review of SNOMED CT use," Journal of the American Medical Informatics Association, vol. 21, no. e1, pp. e11-e19, Feb. 2014.

[11] R. Mudunuri, O. Burgert, and T. Neumuth, "Ontological Modelling of Surgical Knowledge.” GI Jahrestagung, vol. 154, pp. 1044-1054, 2009.

[12] D. Katić, M. Maleshkova, S. Engelhardt, I. Wolf, K. März, L. MaierHein, M. Nolden, M. Wagner, H. Kenngott, B. P. Müller-Stich, R. Dillmann, and S. Speidel, "What does it all mean? Capturing Semantics of Surgical Data and Algorithms with Ontologies," arXiv:1705.07747 [cs], p. 4, May 2017.

[13] Mudunuri R, Neumuth T, Strauß G, Dietz A, Meixensberger J, and Burgert O, "SOCAS - Surgical Ontologies for Computer Assisted Surgery." 2007, p. 481.

[14] D. Katic, C. Julliard, A.-L. Wekerle, H. Kenngott, B. P. Müller-Stich, R. Dillmann, S. Speidel, P. Jannin, and B. Gibaud, "LapOntoSPM: An ontology for laparoscopic surgeries and its application to surgical phase recognition," Int J Comput Assist Radiol Surg, vol. 10, no. 9, pp. 1427 1434, Jun. 2015.

[15] Á. Takács, I. Rudas, and T. Haidegger, "Open-Source Research Platforms and System Integration in Modern Surgical Robotics,' Acta Universitatis Sapientiae Electrical and Mechanical Engineering, vol. 14, no. 6, pp. 20-34, 2015.

[16] J. H. Gennari, M. A. Musen, R. W. Fergerson, W. E. Grosso, M. Crubézy, H. Eriksson, N. F. Noy, and S. W. Tu, "The evolution of Protégé: An environment for knowledge-based systems development," International Journal of Human-Computer Studies, vol. 58, no. 1, pp. 89-123, Jan. 2003.

[17] M. A. Musen, "The Protégé Project: A Look Back and a Look Forward," AI matters, vol. 1, no. 4, pp. 4-12, Jun. 2015.

[18] VLC, "VLC media player, the best Open Source player - VideoLAN," https://www.videolan.org/vlc/index.html, 2017. 
[19] M. Quigley, K. Conley, B. Gerkey, J. Faust, T. Foote, J. Leibs, R. Wheeler, and A. Y. Ng, "ROS: An open-source Robot Operating System," in ICRA Workshop on Open Source Software, vol. 3. Kobe, 2009 , p. 5.

[20] J. Tokuda, L. Ibanez, C. Csoma, P. Cheng, H. Liu, J. Blevins, J. Arata, $\mathrm{X}$. Papademetris, and N. Hata, "Software and hardware integration strategy for image guided therapy using Open IGT Link," in Workshop on Systems and Architectures for Computer Assisted Interventions, MICCAI, 2008.

[21] D. Á. Nagy, I. J. Rudas, and T. Haidegger, "Surgical Data Science, An Emerging Field of Medicine," in IEEE 30th Jubilee Neumann Colloquium, Budapest, November 24-25, 2017, pp. 1-5. 
D. A. Nagy et al. • OntoFlow, a Software Tool for Surgical Workflow Recording 